\title{
Erratum
}

\section{Erratum to "Factors Associated with Long-Term Control of Type 2 Diabetes Mellitus"}

\author{
Mohammed Badedi $\left(\mathbb{D},{ }^{1}\right.$ Yahiya Solan $\left(\mathbb{D},{ }^{2}\right.$ Hussain Darraj $\left(\mathbb{D},{ }^{3}\right.$ Abdullah Sabai $\left(D,{ }^{3}\right.$ \\ Mohamed Mahfouz $\left(\mathbb{D},{ }^{4}\right.$ Saleh Alamodi, ${ }^{5}$ and Abdullah Alsabaani ${ }^{6}$ \\ ${ }^{1}$ Public Health Administration, Jazan Health Affairs, Ministry of Health, Jazan, Saudi Arabia \\ ${ }^{2}$ Diabetes Center, Jazan Health Affairs, Jazan, Saudi Arabia \\ ${ }^{3}$ Jazan Health Affairs, Ministry of Health, Jazan, Saudi Arabia \\ ${ }^{4}$ Faculty of Medicine, Jazan University, Jazan, Saudi Arabia \\ ${ }_{5}^{5}$ Jazan General Directorate of Education, Ministry of Education, Jazan, Saudi Arabia \\ ${ }^{6}$ College of Medicine, King Khalid University, Abha, Saudi Arabia \\ Correspondence should be addressed to Mohammed Badedi; dr.badedi@gmail.com
}

Received 6 February 2019; Accepted 14 February 2019; Published 6 May 2019

Copyright (c) 2019 Mohammed Badedi et al. This is an open access article distributed under the Creative Commons Attribution License, which permits unrestricted use, distribution, and reproduction in any medium, provided the original work is properly cited.

In the article titled "Factors Associated with Long-Term Control of Type 2 Diabetes Mellitus" [1], the less and greater than signs were reversed in the tables and the text. In addition, there were minor errors in Introduction and the format of Table 2.

(1) In Introduction, there was a minor spelling error where the text reading "To the best of our knowledge, this is the first study that has been carried out on patinets with T2DM to identfy factors related to glycemic control in the Jazan region of Saudi Arabia" should be corrected to "To the best of our knowledge, this is the first study that has been carried out on patients with T2DM to identify factors related to glycemic control in the Jazan region of Saudi Arabia."

(2) The last sentence in the "Data Analysis" subsection in the Methods reading "A $P$ value of $>0.05$ was considered to be statistically significant" should be corrected to "A $P$ value of $<0.05$ was considered to be statistically significant."

(3) In the first paragraph of the "Result" section, the sentence reading "Of the total respondents, $74 \%$ had poor glycemic control (HbA1c < 7\%)" should be corrected to "Of the total respondents, 74\% had poor glycemic control (HbA1c > 7\%).”

(4) In Table 1, the category "Duration of diabetes (year)" included reversed less and greater than signs. The corrected table is as shown below.

(5) In Table 2, the less and greater than signs were reversed in many categories and the format of the category "Medication and treatment modalities" should be updated for clarity. The corrected table is as shown below.

(6) In the subsection titled "Logistic Regression Analysis of Factors Associated with HbAlc" in the "Result" section, the text reading "Variables in the regression model included not taking medication $(\mathrm{OR}=4.06$, $P=0.013)$, number of medications (OR $=7.49, P>$ $0.005)$, extended duration of diabetes $(\mathrm{OR}=4.64$, $P=0.001$ ), and low confidence in the ability to control diabetes" should be corrected to "Variables in the regression model included not taking medication $(\mathrm{OR}=4.06, P=0.013)$, number of medications $(\mathrm{OR}=7.49, P<0.005)$, extended duration of 
TABLE 1: Sociodemographic and health risk factors.

\begin{tabular}{|c|c|c|c|c|}
\hline Variable & Categories & $n(\%)$ & HbAlc & $P$ \\
\hline \multirow{3}{*}{ Age (year) } & $28-49$ & $n=87(30.2 \%)$ & 9 & \multirow{3}{*}{.011} \\
\hline & $50-64$ & $n=148(51.4 \%)$ & 8.7 & \\
\hline & $64-83$ & $n=53(18.4 \%)$ & 7.7 & \\
\hline \multirow{2}{*}{ Sex } & Male & $n=145(50.3 \%)$ & 8.5 & \multirow{2}{*}{.083} \\
\hline & Female & $n=143(49.7 \%)$ & 8.9 & \\
\hline \multirow{4}{*}{ Marital status } & Divorced & $n=7(2.4 \%)$ & 11.5 & \multirow{4}{*}{.005} \\
\hline & Single & $n=16(5.6 \%)$ & 9.5 & \\
\hline & Widowed & $n=36(12.5 \%)$ & 9.4 & \\
\hline & Married & $n=229(79.5 \%)$ & 8.5 & \\
\hline \multirow{6}{*}{ Education level } & Illiterate & $n=36(12.5 \%)$ & 9.2 & \multirow{6}{*}{.032} \\
\hline & Read and write & $n=33(11.5 \%)$ & 9.1 & \\
\hline & Elementary school level & $n=41(14.2 \%)$ & 8.9 & \\
\hline & Intermediate school level & $n=42(14.6 \%)$ & 8.8 & \\
\hline & Secondary school level & $n=57(19.8 \%)$ & 8.2 & \\
\hline & University level & $n=79(29.4 \%)$ & 8.1 & \\
\hline \multirow{6}{*}{ Occupation } & Unemployed & $n=8(2.1 \%)$ & 8.8 & \multirow{6}{*}{.691} \\
\hline & Employed & $n=105(36.5 \%)$ & 8.3 & \\
\hline & Retired & $n=67(23.3 \%)$ & 8.7 & \\
\hline & Homemaker & $n=103(35.8 \%)$ & 8.9 & \\
\hline & Businessman & $n=4(1.4 \%)$ & 8.9 & \\
\hline & Disabled & $n=3(1 \%)$ & 7.6 & \\
\hline \multirow{3}{*}{ Smoking history } & Smoker & $n=63(21.9 \%)$ & 9.4 & \multirow{3}{*}{.031} \\
\hline & Ex-smoker & $n=2(0.7 \%)$ & 8.6 & \\
\hline & Nonsmoker & $n=223(77.4 \%)$ & 8.5 & \\
\hline \multirow{2}{*}{ Duration of diabetes (year) } & $\geq 7$ & $n=166(42.4 \%)$ & 9.1 & \multirow{2}{*}{$<.001$} \\
\hline & $<7$ & $n=122(57.6 \%)$ & 7.5 & \\
\hline \multirow{4}{*}{$\begin{array}{l}\text { Other chronic diseases or diabetes } \\
\text { complications }\end{array}$} & Irritable bowel syndrome (IBS) & $n=9(3.1 \%)$ & 11.5 & \multirow{4}{*}{.020} \\
\hline & Hypertension (HTN) & $n=162(56.2)$ & 8.8 & \\
\hline & Asthma & $n=6(2.1 \%)$ & 8.8 & \\
\hline & $\begin{array}{l}\text { No other chronic disease or } \\
\text { diabetes complications }\end{array}$ & $n=111(38.6 \%)$ & 8.5 & \\
\hline
\end{tabular}


TABLE 2: Self-care behavior's adherence and HbA1c.

\begin{tabular}{|c|c|c|c|c|}
\hline Variable & Categories & $n(\%)$ & HbA1c (\%) & $P$ \\
\hline \multirow{2}{*}{ Following a meal plan } & Low adherence & $n=232(80.6 \%)$ & 9.0 & \multirow{2}{*}{$<.001$} \\
\hline & High adherence & $n=56(19.4 \%)$ & 7.3 & \\
\hline \multirow{2}{*}{ Taking medications } & Low adherence & $n=89(30.9 \%)$ & 9.2 & \multirow{2}{*}{.001} \\
\hline & High adherence & $n=199(69.1 \%)$ & 8.2 & \\
\hline \multirow{2}{*}{ Exercising } & Low adherence & $n=121(42 \%)$ & 8.8 & \multirow{2}{*}{.310} \\
\hline & High adherence & $n=167(58 \%)$ & 8.6 & \\
\hline \multirow{2}{*}{ Testing blood glucose } & Low adherence & $n=146(50.7 \%)$ & 8.9 & \multirow{2}{*}{.301} \\
\hline & High adherence & $n=142(49.3 \%)$ & 8.6 & \\
\hline \multirow{2}{*}{ Following a meal plan and taking medication } & Low adherence & $n=80(27.8 \%)$ & 9.4 & \multirow{2}{*}{$<.001$} \\
\hline & High adherence & $n=47(16.3 \%)$ & 7.0 & \\
\hline \multirow{2}{*}{ Following a meal plan and exercising regularly } & Low adherence & $n=105(36.5 \%)$ & 9.0 & \multirow{2}{*}{$<.001$} \\
\hline & High adherence & $n=40(13.9 \%)$ & 7.4 & \\
\hline \multirow{2}{*}{$\begin{array}{l}\text { Following a meal plan, taking medication, } \\
\text { exercising, and testing blood glucose }\end{array}$} & Low adherence & $n=37(12.8 \%)$ & 10.1 & \multirow{2}{*}{$<.001$} \\
\hline & High adherence & $n=26(9 \%)$ & 6.9 & \\
\hline \multirow{2}{*}{ Number of medications } & $>4$ & $n=136(47.2 \%)$ & 9.5 & \multirow{2}{*}{.001} \\
\hline & $\leq 4$ & $n=152(52.8 \%)$ & 7.4 & \\
\hline \multirow{2}{*}{ Treatment modalities } & Oral antidiabetic agents alone & $n=229(79.5 \%)$ & 8.7 & \multirow{2}{*}{.740} \\
\hline & Oral antidiabetic agents and insulin & $n=59(20.5 \%)$ & 8.7 & \\
\hline \multirow{4}{*}{ Medication and treatment modalities } & $\begin{array}{l}\text { Low medication adherence-oral } \\
\text { antidiabetic agents with insulin }\end{array}$ & $n=26(9 \%)$ & 9.5 & \multirow{4}{*}{.001} \\
\hline & $\begin{array}{l}\text { Low medication adherence-oral } \\
\text { antidiabetic agents alone }\end{array}$ & $n=60(20.8 \%)$ & 9.2 & \\
\hline & $\begin{array}{l}\text { High medication adherence-oral } \\
\text { antidiabetic agents alone }\end{array}$ & $n=169(58.7 \%)$ & 8.2 & \\
\hline & $\begin{array}{l}\text { High medication adherence-oral } \\
\text { antidiabetic agents with insulin }\end{array}$ & $n=33(11.5 \%)$ & 8.1 & \\
\hline
\end{tabular}


TABLe 4: Anthropometrics and HbAlc levels.

\begin{tabular}{|c|c|c|c|c|}
\hline Variable & Categories & $n(\%)$ & $\mathrm{HbAlc}$ & $P$ \\
\hline \multirow{2}{*}{ Family provides help and support } & Lesser extent (a little) & $n=76(26.4 \%)$ & 9.4 & \multirow{2}{*}{.002} \\
\hline & Greater extent (a lot) & $n=212(73.6 \%)$ & 8.4 & \\
\hline \multirow{2}{*}{ Physician-patient relationship } & Lesser extent (seldom) & $n=41(14.2 \%)$ & 10.6 & \multirow{2}{*}{$<.001$} \\
\hline & Greater extent (often) & $n=247(85.8 \%)$ & 8.5 & \\
\hline \multirow{2}{*}{ Knowledge towards diabetes } & Lesser extent & $n=136(47.2 \%)$ & 8.9 & \multirow{2}{*}{.020} \\
\hline & Greater extent & $n=152(52.8 \%)$ & 8.5 & \\
\hline \multirow{2}{*}{ Confidence in ability to manage self-care behaviors } & Not confident & $n=159(55.2 \%)$ & 8.9 & \multirow{2}{*}{.001} \\
\hline & Confident & $n=129(44.8 \%)$ & 8.5 & \\
\hline \multirow{3}{*}{ Physical health } & $<40$ & $n=57(19.8 \%)$ & 11.8 & \multirow{3}{*}{$<.001$} \\
\hline & $40-50$ & $n=93(32.3 \%)$ & 9.12 & \\
\hline & $>50$ & $n=138(47.9 \%)$ & 7.50 & \\
\hline \multirow{3}{*}{ Depression } & Major depression & $n=41(14.2 \%)$ & 11.3 & \multirow{3}{*}{$<.001$} \\
\hline & Atypical depression & $n=63(21.9 \%)$ & 8.70 & \\
\hline & No depression & $n=184(63.9 \%)$ & 7.85 & \\
\hline \multirow{3}{*}{ Stressful life events } & High risk $>300$ & $n=24(8.3 \%)$ & 11.8 & \multirow{3}{*}{$<.001$} \\
\hline & Moderate risk 150-300 & $n=45(15.6 \%)$ & 8.90 & \\
\hline & Low risk $<150$ & $n=219(76 \%)$ & 8.00 & \\
\hline \multirow{2}{*}{ Blood pressure (BP) (mmHg) } & High blood pressure & $n=127(44.1 \%)$ & 9.0 & \multirow{2}{*}{.073} \\
\hline & Normal blood pressure & $n=161(55.9 \%)$ & 8.5 & \\
\hline \multirow{4}{*}{ Body mass index (BMI) $\left(\mathrm{kg} / \mathrm{m}^{2}\right)$} & Obese & $n=134(46.5 \%)$ & 8.9 & \multirow{4}{*}{.01} \\
\hline & Overweight & $n=107(37.2 \%)$ & 8.7 & \\
\hline & Normal weight & $n=44(15.3 \%)$ & 7.9 & \\
\hline & Underweight & $n=3(1 \%)$ & 6.3 & \\
\hline \multirow{2}{*}{ Cholesterol (mg/dl) } & Blood cholesterol $\geq 200$ & $n=117(40.6 \%)$ & 9.2 & \multirow{2}{*}{$<.001$} \\
\hline & Blood cholesterol $<200$ & $n=171(59.4 \%)$ & 8.1 & \\
\hline \multirow{2}{*}{ High-density lipoprotein (HDL) (mg/dl), male } & Low $\mathrm{HDL}<40$ & $n=80(55.2 \%)$ & 9.1 & \multirow{2}{*}{$<.001$} \\
\hline & High $\mathrm{HDL} \geq 40$ & $n=65(44.8 \%)$ & 7.6 & \\
\hline \multirow{2}{*}{ High-density lipoprotein (HDL) (mg/dl), female } & Low $\mathrm{HDL}<50$ & $n=94(65.7 \%)$ & 9.1 & \multirow{2}{*}{.027} \\
\hline & High HDL $\geq 50$ & $n=49(34.3 \%)$ & 7.8 & \\
\hline \multirow{2}{*}{ Low-density lipoprotein (LDL) (mg/dl) } & High $\mathrm{LDL} \geq 100$ & $n=198(68.8 \%)$ & 8.8 & \multirow{2}{*}{.026} \\
\hline & Low $\mathrm{LDL}<100$ & $n=90(31.2 \%)$ & 8.2 & \\
\hline \multirow{2}{*}{ Triglyceride (TG) (mg/dl) } & High $\mathrm{TG} \geq 150$ & $n=116(40.3 \%)$ & 9.1 & \multirow{2}{*}{$<.01$} \\
\hline & Low $\mathrm{TG}<150$ & $n=172(59.7 \%)$ & 8.4 & \\
\hline
\end{tabular}

TABLE 5: Regression model for factors associated with HbA1c.

\begin{tabular}{|c|c|c|c|}
\hline Variable & Categories & OR ( $95 \%$ confidence interval) & $P$ \\
\hline \multirow{2}{*}{ Taking medication } & Low adherence & \multirow{2}{*}{$4.06(1.34,12.27)$} & \multirow{2}{*}{.013} \\
\hline & High adherence & & \\
\hline \multirow{2}{*}{ Number of medications } & $>4$ & \multirow{2}{*}{$7.49(3.45,16.26)$} & \multirow{2}{*}{$<.005$} \\
\hline & $\leq 4$ & & \\
\hline \multirow{2}{*}{ Duration of diabetes (year) } & $\geq 7$ & \multirow{2}{*}{$4.64(1.85,11.67)$} & \multirow{2}{*}{.001} \\
\hline & $<7$ & & \\
\hline \multirow[t]{2}{*}{ Confidence in ability to manage self-care behaviors } & Not confident & \multirow[t]{2}{*}{$4.01(1.52,10.63)$} & \multirow[t]{2}{*}{.005} \\
\hline & Confident & & \\
\hline
\end{tabular}


diabetes $(\mathrm{OR}=4.64, P=0.001)$, and low confidence in the ability to control diabetes."

(7) In Table 4, the less and greater than signs were reversed in many categories. The corrected table is as shown below.

(8) In Table 5, the less and greater than signs were reversed in many categories. The corrected table is as shown below.

\section{References}

[1] M. Badedi, Y. Solan, H. Darraj et al., "Factors associated with long-term control of type 2 diabetes mellitus," Journal of Diabetes Research, vol. 2016, Article ID 2109542, 8 pages, 2016. 


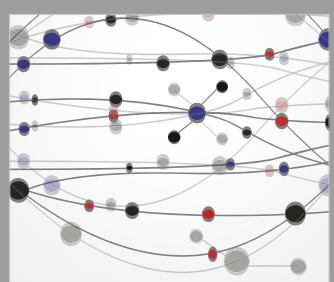

The Scientific World Journal
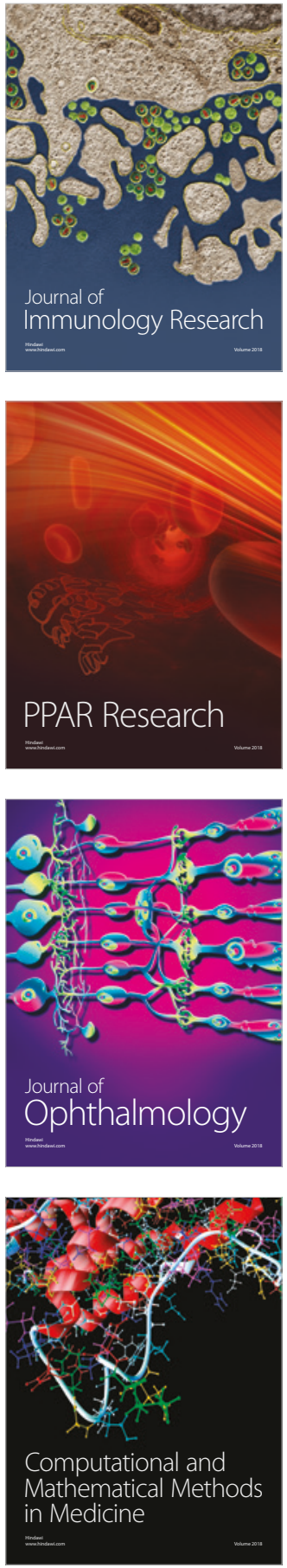

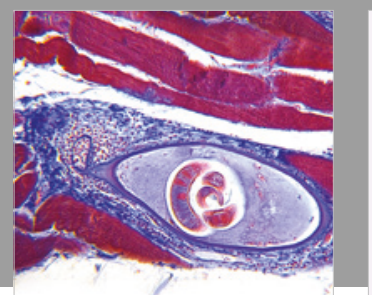

Gastroenterology Research and Practice

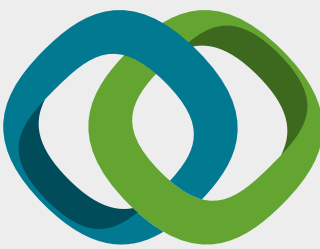

\section{Hindawi}

Submit your manuscripts at

www.hindawi.com
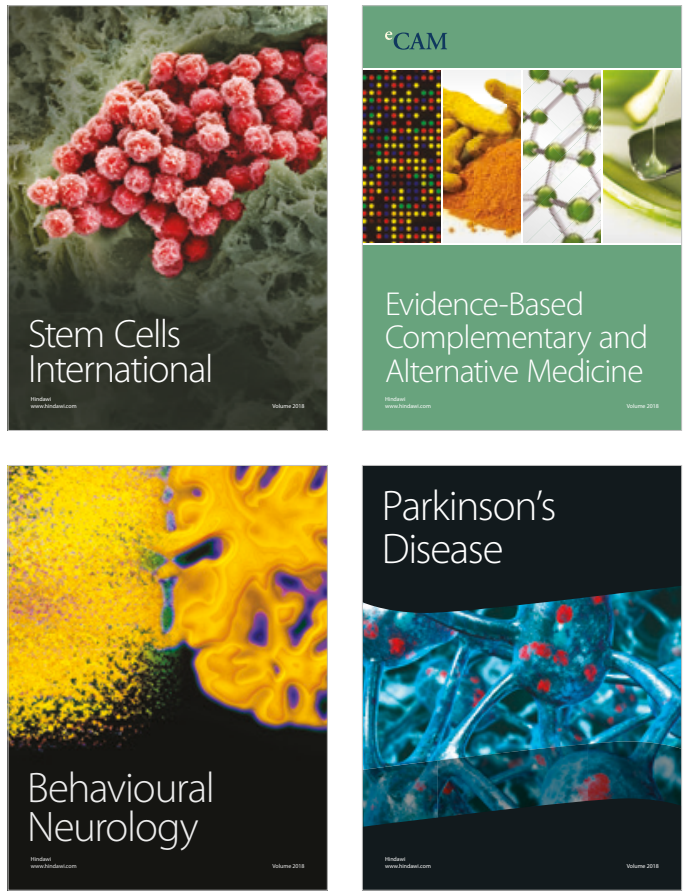

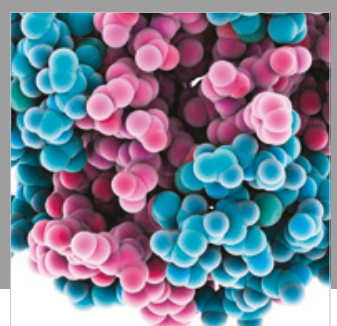

ournal of

Diabetes Research

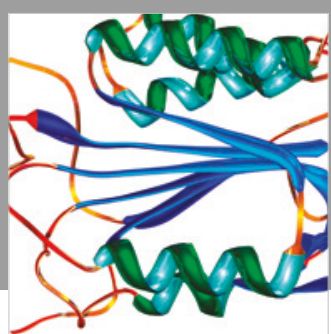

Disease Markers
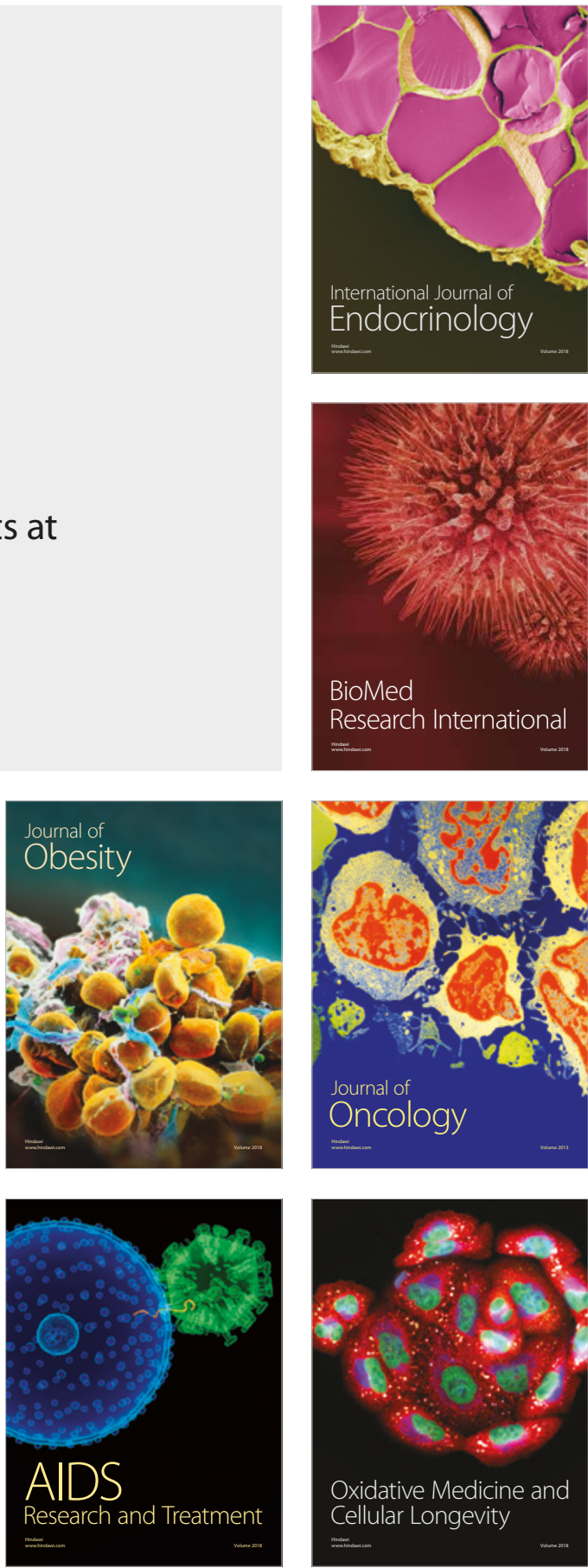